\title{
Mixed convection of micropolar fluid in a vertical double-passage channel
}

\author{
J. C. Umavathi \\ Department of Mathematics, Gulbarga University, Gulbarga-585 106, Karnataka, INDIA. \\ E-mail: jc_uma11@yhaoo.com
}

\begin{abstract}
The effect of the presence of a thin perfectly conductive baffle on the fully developed laminar mixed convection in a vertical channel containing micropolar fluid is analyzed. The channel has different constant wall temperatures. Analytical expressions for velocity and microrotation velocity are obtained. The solutions are evaluated numerically and shown graphically for the ratio of Grashof number to Reynolds number, material parameter and the baffle position on the velocity and microrotation velocity. It is found that the ratio of Grashof number to Reynolds number promotes the velocity whereas it suppresses the microrotation velocity and the material parameter is invariant on velocity but it promotes the microrotation velocity. Flow reversal can also be found in the double-passage channel with suitable choice of the ratio of Grashof number to Reynolds number.
\end{abstract}

Keywords: Mixed convection; micropolar; double-passage.

DOI: http://dx.doi.org/10.4314/ijest.v3i8.17

\section{Introduction}

The physical mechanisms of heat, mass, and momentum transport in small-scale units may differ significantly from those in macro scale equipment (Popautsky et al., 1999 and Shu, 2004). Fundamental and applied investigations of micro scale phenomena in fluid mechanics are motivated by developments in the areas of biological molecular machinery, atherogenesis, microcirculation, and micro fluidics. At scales larger than a micron, the fluid can be treated as a continuum, and the flow is governed by the NavierStokes equation. The continuum model assumes that the properties of the material vary continuously throughout the flow domain. In Newtonian continuum mechanics, the fluid is modeled as a dense aggregate of particles, possessing mass and translational velocity. However, the field equation, such as the Navier-Stokes equation, does not account for the rotational effects of the fluid micro-constituents. In the theory of micropolar fluids (Eringen, 2001), rigid particles contained in a small volume element can rotate about the centered of the volume element. The rotation is described by an independent micro-rotation vector. Micropolar fluids can support body couples and exhibit microrotational effects. The theory of micropolar fluids has shown promise for predicting fluid behavior at microscale. Papautsky et al. (1999) found that a numerical model for water flow in microchannels based on the theory of micropolar fluids gave better predictions of experimental results than those obtained using the NavierStokes equation. Micropolar fluids can model anisotropic fluids, liquid crystals with rigid molecules, magnetic fluids, clouds, clouds with dust, muddy fluids, and some biological fluids (Eringen, 2001).

The theory of microfluids, as developed by Eringen (1964) has been a field of active research for the last few decades as this class of fluids represents mathematically many industrially important fluids like paints, blood, body fluids, polymers, colloidal fluids and suspension fluids. In this theory, the local effects arising from the microstructure and intrinsic motion of the fluid elements are taken into account. Such fluids can be subjected to surface and body couples in which the material points in a volume element can undergo motions about centre of mass along with deformation. The problem of simple microfluid contains a system of 19 equations with 19 unknowns so that it becomes difficult to find a solution. A subclass of these fluids introduced by Eringen (1966) is the micropolar fluids, which exhibit the microrotational effects and microrotational inertia. Under these assumptions, deformation of the fluid microelements is ignored. An excellent review of micropolar fluids and their applications was given by Ariman et al. (1974) and Hoyt and Fabula (1964) has shown experimentally that the fluids containing minute polymeric additives 
indicate considerable reduction of the skin friction (about 25-30\%), a concept which can be well explained by the theory of micropolar fluids. Chamkha et al. (2002) found the numerical and analytical solutions for fully developed free convection of a micropolar fluid in a vertical channel. El-Hakien (2004) found the similarity solutions for free convection in a micropolar fluid with thermal dispersion and internal heat generation. Then Cheng, $(2006,2008)$ studied the flow nature of micropolar fluid in a vertical channel and near a sphere. Umavathi et al. $(2008,2010)$ and Prathap Kumar et al. (2010) analyzed flow and heat transfer of a micropolar fluid. Anwar et al. (2011) analyzed the numerical simulation in micropolar fluid dynamics. Analytical and semi analytical solutions for micropolar fluid in a porous channel was recently studied by Rashidi et al. (2010, 2011).

The free convective flow of fluids with microstructure is an interesting area of research including liquid crystals, dilute solutions of polymer fluids and many types of suspensions. A situation where both the natural and forced convection effects are of comparable order is called mixed or combined convection. Mixed convection flows in channels and ducts find applications in nuclear reactors, heat exchangers etc. and have been studied by various authors namely Hsu and Chen (1996) and Rama Bhargava et al. (2003). The buoyancy forces may aid or oppose the forced flow causing an increase or decrease in the heat transfer. The most commonly used technique for internal cooling enhancement is the placement of periodic ribs. Ribs are generally mounted on the heat transfer surface, which disturbs the boundary layer growth and enhances the heat transfer between the surface and the fluid. In addition to ribs and impingement, a third kind of common internal cooling enhancement technique is the placement of internal flow swirls, tape twisters, or baffles. The swirl insert and tape twister techniques create a significant amount of bulk flow disturbance, and the pressure drop penalties are much higher compared to the gain in heat transfer coefficient. Baffles also create bulk flow disturbance, but unlike tapers or swirls, baffles are discrete objects. Therefore, the flow disturbance created by baffles may be localized.

Dutta and Dutta (1998) first reported the enhancement of heat transfer with inclined solid and perforated baffles. Later Dutta and Hossian (2005) did the experimental study to analyse the local heat transfer characteristics in a rectangular channel with inclined solid and perforated baffles. Experimental observations and numerical simulations by Howes et al. (1991) and Howes and Shardlow (1997) showed that oscillatory flows in a rectangular channel with a series of baffles can result in vigorous shedding and efficient mixing. Salah El-Din (1994, 1997, 1998, 2002) published a series of papers on mixed convection in a vertical channel by introducing a perfectly conducting baffle. Mousavi and Hooman (2006) studied numerically the fluid flow and heat transfer in the entrance region of a two dimensional horizontal channel with isothermal walls and with staggered baffles. Heat transfer enhancement in a heat exchanger tube by installing a baffle was reported by Nasiruddin and Siddiqui (2007). They found that the average Nusselt number for the two baffles case is $20 \%$ higher than the one baffle case and $82 \%$ higher than the no baffle case. In 2008 Hakeem et al. inserted mutually orthogonal heat generating baffles in a square cavity and conclude that the fluid flow and temperature fields strongly depend on location of the baffles. Recently Prathatp Kumar et al. (2011a, b) analyzed the flow nature by inserting a vertical baffle for viscoelastic fluid (Walters B' model) through vertical wavy channel. It is observed from the literature that introducing a baffle will increase the rate of heat transfer. Hence the results obtained by introducing baffle or fins or ribs can be used in practical applications which requires enhancement of heat transfer.

Keeping in view the application of micropolar fluid in various fields as mentioned, the objective of the present work is to study the effect of baffle on fully developed laminar mixed convection in a vertical double-passage channel for micropolar fluid.

\section{Problem Formulation}

The channel shown in Figure 1 is divided into two passages by means of a perfectly conductive and thin baffle. Consideration is given to a laminar, incompressible, steady flow of micropolar fluid in the channel. The fluid enters the channel with a uniform upward vertical velocity and constant temperature. The channel walls are subjected to different constant temperatures, which are higher than that at the entrance. The fluid properties are assumed to be constant except for the buoyancy term of the momentum equation. The $x$-axis is chosen parallel to the gravitational field, but with opposite direction and $y$-axis is transverse to the channel walls.

For fully developed flow it is assumed that the transverse velocity and temperature gradient in the axial direction are zero. With the assumptions made above, the governing equations of motion and energy reduce to (Eringen, 1966, Ahmadi, 1976).

$$
\begin{gathered}
\rho_{0} g \beta\left(T_{i}-T_{r}\right)-\frac{d p_{i}}{d x}+(\mu+\kappa) \frac{d^{2} u_{i}}{d y^{2}}+\kappa \frac{d \bar{N}_{i}}{d y}=0 \\
\alpha \frac{d^{2} \bar{N}_{i}}{d y^{2}}-2 \kappa \bar{N}_{i}-\kappa \frac{d u_{i}}{d y}=0 \\
\frac{d^{2} T_{i}}{d y^{2}}=0
\end{gathered}
$$

The boundary conditions on velocity, microrotation velocity and temperature are

$$
y=0: \quad u_{1}=0 ; \quad \bar{N}_{1}=0 ; \quad T_{1}=T_{c}
$$




$$
\begin{gathered}
y=b^{*}: \quad u_{1}=u_{2}=0 ; \quad \bar{N}_{1}=\bar{N}_{2}=0 ; \quad T_{1}=T_{2} ; \quad \bar{N}_{2}=0 ; \quad T_{1}=0 ; \quad \frac{d T_{1}}{d y}=\frac{d T_{2}}{d y} \\
y=b: \quad T_{h}
\end{gathered}
$$

where $x$ and $y$ are axial and transverse coordinate respectively, ( $x=0$ is the duct entrance and $y=0$ is the flat wall), $u$ is the axial velocity, $T$ is the temperature, $\bar{N}$ is the microrotation component of the micropolar fluid normal to $(x, y)$ - plane, $T_{c}$ is the temperature on the cold wall (i.e. at $y=0$ ). $T_{h}$ is the temperature of the hot wall (i.e. at $y=h$ ) and $\rho_{0}, g, \beta, \mu, \kappa$ and $\alpha$ are the density, gravitational acceleration, coefficient of thermal expansion, dynamic viscosity, vortex viscosity and spin gradient viscosity respectively. The subscript $i$ denotes either Stream 1 or Stream 2. Equation (1) has been written according to the Boussinesq approximation and that in (3) the viscous dissipation is considered negligible.

The average of the walls temperatures is chosen as the reference temperature,

i.e., $\quad T_{r}=\frac{T_{c}+T_{h}}{2}$

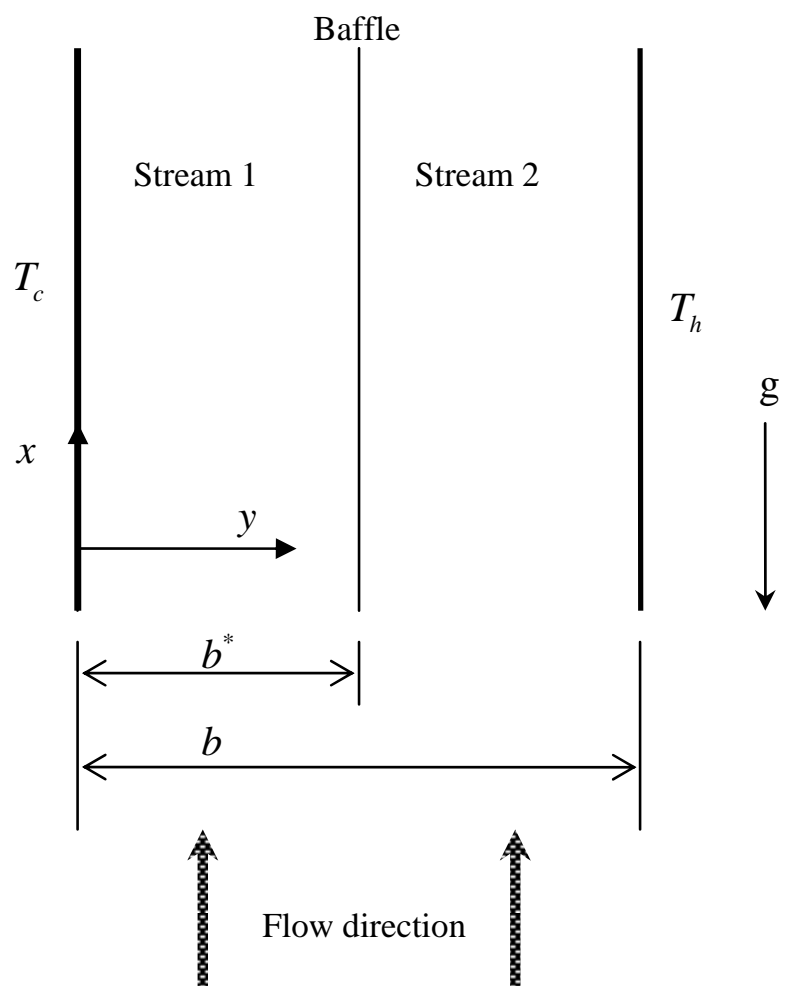

Figure 1: Physical model and the coordinate system.

The governing Eqs. (1) to (3) along with boundary conditions given in Eq. (4) can be expressed in dimensionless form using the following transformations

$$
\begin{gathered}
X=\frac{x}{b \operatorname{Re}} ; Y=\frac{y}{b} ; Y^{*}=\frac{y}{b^{*}} ; U_{i}=\frac{u_{i}}{u_{r}} ; \theta_{i}=\frac{T_{i}-T_{r}}{T_{h}-T_{c}} ; P_{i}=\frac{p_{i}}{\rho_{r} u_{r}^{2}} ; \bar{N}_{i}=\frac{u_{r}}{b} N_{i} ; \alpha=j\left(\mu+\frac{\kappa}{2}\right) \\
G r=\frac{g \beta\left(T_{h}-T_{c}\right) b^{3}}{v^{2}} ; \operatorname{Re}=\frac{u_{r} b}{v} ; k=\mu K ; \lambda=\frac{G r}{\operatorname{Re}}
\end{gathered}
$$

where $j$ is the microinertia density. We follow the work of many recent authors by assuming that $\alpha=j\left(\mu+\frac{\kappa}{2}\right)=\mu\left(1+\frac{K}{2}\right) j$ where $K=\frac{\kappa}{\mu}$ is the material parameter. This assumption is invoked to allow the field of equations to predict the correct behavior in the limiting case when the microstructure effects become negligible and the total spin $\bar{N}$ reduces to the angular velocity (Kumar et al., 2011). 
The reference velocity $u_{r}$ is defined by

$$
u_{r}=\frac{1}{b} \int_{0}^{b} u d y
$$

The governing Eqs. (1) - (3) along with boundary conditions (4) using (6) and (7) become

$$
\begin{gathered}
(1+K) \frac{d^{2} U_{i}}{d Y^{2}}=-\lambda \theta_{i}+\frac{d P_{i}}{d X}-K \frac{d N_{i}}{d Y} \\
\left(1+\frac{K}{2}\right) \frac{d^{2} N_{i}}{d Y^{2}}-2 K N_{i}-K \frac{d U_{i}}{d Y}=0 \\
\frac{d^{2} \theta_{i}}{d Y^{2}}=0
\end{gathered}
$$

Subject to boundary conditions

$$
\begin{aligned}
& Y=0: \quad U_{1}=0 ; \quad N_{1}=0 ; \quad \theta_{1}=-\frac{1}{2} \\
& Y=Y^{*}: \quad U_{1}=U_{2}=0 ; \quad N_{1}=N_{2}=0 ; \quad \theta_{1}=\theta_{2} ; \quad \frac{d \theta_{1}}{d Y}=\frac{d \theta_{2}}{d Y} \\
& Y=1: \quad U_{2}=0 ; \quad N_{2}=0 ; \quad \theta_{2}=\frac{1}{2} .
\end{aligned}
$$

where $Y^{*}$ represents the position of the baffle. The boundary conditions on temperature are Dirichlet boundary conditions. However solutions of Eq. (11) can also be found using Robin boundary conditions or boundary conditions of third kind (see Zanchini, 1998). The pressure gradient in Eq. (9) is considered as $\frac{d P_{i}}{d X}=\gamma_{i}$ which is a constant. Since the pressure gradients in both passages can be controlled at one's disposal, the respective flow rate is assumed to be distributed proportional to the area of each passage. Therefore conservation of mass considered at any cross section of the channel passage gives

$$
\int_{0}^{Y^{*}} U_{1} d Y=Y^{*} ; \quad \int_{Y^{*}}^{1} U_{2} d Y=1-Y^{*} ; \quad \int_{0}^{Y^{*}} U_{1} d Y+\int_{Y^{*}}^{1} U_{2} d Y=1
$$

\section{Closed form Solutions}

Equations (9) to (11) with boundary conditions (12) can be solved exactly. The expressions for the non-dimensional temperature, velocity, and microrotational velocity are given by

Stream 1

$$
\begin{gathered}
\theta_{1}=Y-\frac{1}{2} \\
U_{1}=f_{1} \sinh (\sqrt{\tau} Y)+f_{2} \cosh (\sqrt{\tau} Y)+f_{3} Y^{3}+f_{4} Y^{2}+c_{1} Y+c_{2} \\
N_{1}=d_{1} \cosh (\sqrt{\tau} Y)+d_{2} \sinh (\sqrt{\tau} Y)+l_{2} Y^{2}+l_{4} Y+l_{5}
\end{gathered}
$$

Stream 2

$$
\begin{gathered}
\theta_{2}=Y-\frac{1}{2} \\
U_{2}=f_{5} \sinh (\sqrt{\tau} Y)+f_{6} \cosh (\sqrt{\tau} Y)+f_{7} Y^{3}+f_{8} Y^{2}+c_{3} Y+c_{4} \\
N_{2}=d_{3} \cosh (\sqrt{\tau} Y)+d_{4} \sinh (\sqrt{\tau} Y)+l_{2} Y^{2}+l_{6} Y+l_{7}
\end{gathered}
$$

The method of finding analytical solutions is to integrate Eq. (9) and substitute in Eq. (10). Integrating Eq. (9) once will give rise to $\frac{d U_{1}}{d Y}=\frac{1}{(1+K)}\left(-\lambda\left(\frac{Y^{2}}{2}-\frac{Y}{2}\right)+\gamma_{1} y-K N_{1}\right)+A_{1}$ where $A_{1}$ is the integrating constant to be evaluated after finding the solutions for $U_{1}$ and $N_{1}$. Substituting the above expression for $\frac{d U_{1}}{d Y}$ in Eq. (10) will reduce the differential Eq. (10) for $N_{1}$ only. Hence integrating twice Eq.(10) will give solution for $N_{1}$. Differentiating this expression for $N_{1}$ once and substituting in Eq. (9) 
will result the differential Eq. (9) in $U_{1}$ only. Integrating twice Eq. (9) will result for the solution of $U_{1}$. The same procedure is repeated to find out the solution for $U_{2}$ and $N_{2}$. Substituting $U_{i}$ in (13) gives the constants $\gamma_{1}$ and $\gamma_{2}$. Finding the solutions of $U_{i}$ and $N_{i}$ is similar to Chamkha et al. (2002) and finding the constants $\gamma_{1}$ and $\gamma_{2}$ is similar to Salah El-Din (2002).

The constants appeared in the above equations are given in the Appendix section.

\section{Solutions in the Newtonian case}

The solutions of Eqs. (9) to (11) become

$$
\begin{gathered}
\theta_{i}=Y_{i}-\frac{1}{2}, \\
U_{1}=\frac{1}{2} \gamma_{1}\left(Y-Y^{*}\right) Y-\frac{\lambda}{12}\left(2 Y^{2}-3 Y-2 Y^{* 2}+3 Y^{*}\right) Y, \\
U_{2}=\frac{(1-Y)}{12}\left(Y^{*}-Y\right)\left(6 W p \gamma_{1}+\lambda\left(1-2 Y^{*}-2 Y\right)\right) .
\end{gathered}
$$

Similar to single-passage channel flow, Eq. (20) reveals that the temperature profile is linear for double-passage channel flow if the fluid is purely viscous, whereas the fluid velocity profiles are both polynomials of degree three.

Substitution of Eqs. (21) and (22) into Eq. (13) leads to

$$
\frac{W p}{12} \gamma_{1}\left(Y^{*}-1\right)^{3}-\frac{\gamma_{1}}{12} Y^{* 3}+\frac{\lambda}{24} Y^{*}\left(2 Y^{*}-1\right)\left(Y^{*}-1\right)=1
$$

Equation (23) gives the relation between pressure gradient in stream- $1 \gamma_{1}$, ratio of pressure gradient $W p\left(=\gamma_{1} / \gamma_{2}\right), \lambda$ and the baffle position $Y^{*}$.

The condition for occurrence of flow reversal in stream-1 is given by

$$
\left.\frac{d U_{1}}{d Y}\right|_{Y=0}=\frac{\gamma_{1}}{2} Y^{*}-\lambda\left(\frac{Y^{* 2}}{6}-\frac{Y^{*}}{4}\right)=0,
$$

and, therefore the corresponding relation between $\gamma_{1}, \lambda$ and $Y^{*}$ can be obtained

$$
\lambda=\frac{6 \gamma_{1}}{2 Y^{*}-3} \text {. }
$$

We note that the pressure gradient ratio $W p$ does not enter into Eq. (25) directly but via Eq. (23).

Combining Eqs. (23) and (25) gives the critical value of $\lambda$ at which the flow reversal occurs and it is given by

$$
\lambda_{c}=\frac{72}{\left(3-2 Y^{*}\right)\left(\left(1-Y^{*}\right)^{3} W p+Y^{* 3}\right)+3 Y^{*}\left(1-2 Y^{*}\right)\left(1-Y^{*}\right)} .
$$

Putting $Y^{*}=1$, the single-passage channel case, in Eq. (26) yields the condition for the occurrence of flow reversal

$$
\lambda_{c}=72 \text {, }
$$

which is only one-fourth of the value provided by Barletta (1998) whose $\lambda=288$ for single vertical channel flow. This is owing to the fact that the length scale used by Barletta (1998) is $2 b$ rather than $b$ in the present study. In this case, the corresponding value of $\gamma_{1}$ is -12 .

Putting $Y^{*}=1 / 2$ in Eqs. (25) and (26), the critical $\lambda$ and $\gamma_{1}$ can be obtained for an equi-partitioned double-passage channel:

$$
\begin{aligned}
& \gamma_{1}=\frac{-96}{1+W p}, \\
& \lambda_{c}=\frac{288}{1+W p} .
\end{aligned}
$$

Hence, using $W p=1$ in Eq. (28), the pressure gradients are

$$
\gamma_{1}=\gamma_{2}=-48
$$

which are the same as that given by Salah El-Din (2002). Under this condition the value of $\lambda$ is 144 for the condition of flow reversal in stream-1. 


\section{Results and discussion}

The object of the present study is to examine the characteristics of mixed convection of a micropolar fluid in a vertical channel containing a thin conducting baffle. Closed form solutions are obtained for velocity and microrotation velocity. The governing Eqs. (9) and (10) shows that the solutions depend on three parameters namely buoyancy-to-inertia force $\lambda$, material parameter $K$ and pressure gradients $\gamma_{i}$. When the parameter $\lambda$ exceeds threshold value, flow reversal may occur causing downward flow along the cool and hot wall for values of $\lambda>0$ or $\lambda<0$. The developments of velocity and microrotation velocity profiles for values $Y^{*}=0.2,0.5,0.8$ are shown in Figures 2 to 5 respectively.
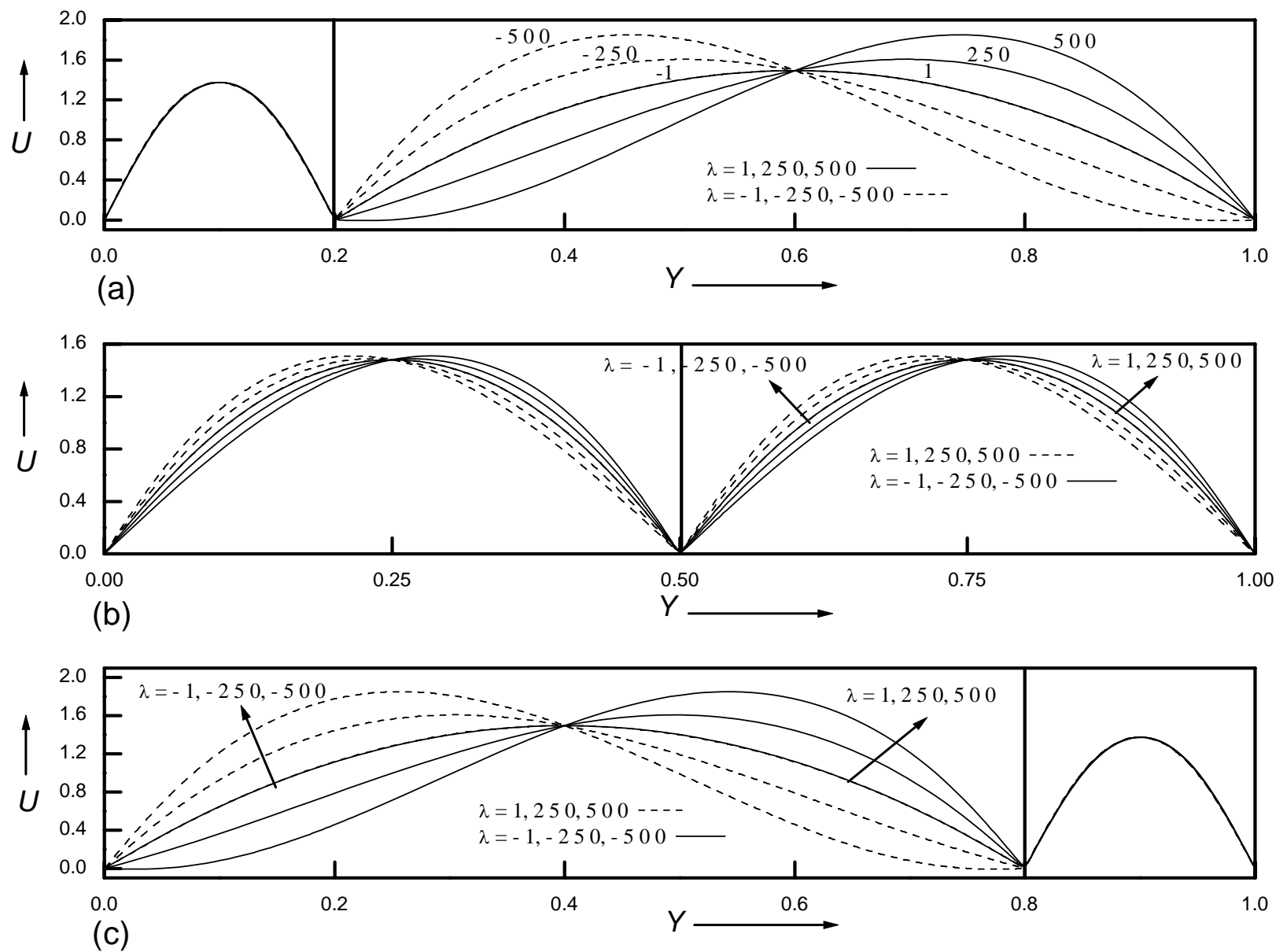

Figure 2: Velocity profiles for varies $\mathrm{Y}^{*}$ with $\lambda= \pm(1,250,500)$ and $\mathrm{K}=2$, a) $\mathrm{Y}^{*}=0.2$, b) $\mathrm{Y}^{*}=0.5$ and c) $\mathrm{Y}^{*}=0.8$.

The effect of buoyancy-to-inertia force $\lambda$ on velocity and micro rotational velocity for various baffle positions are displayed in Figures 2 and 3 respectively with material parameter $K=2$. For each graph the maximum point of the velocity profile moves to the right (hotter) wall for increasing values of $\lambda>0$ and thus the velocity decreases near the left (colder) wall. On the contrary the maximum point of the velocity profile moves to the left (colder) wall for increasing values of $\lambda<0$ and thus the velocity decreases near the right (hotter) wall. The larger the value of $\lambda>0$, stronger the upward velocity. Especially for sufficient large values of $\lambda>0$, the flow reversal is found near the left wall. This is owing to the fact that the buoyancy force is strengthened so as to assist the upper flow in stream-2 for increasing $\lambda$ near the hot wall, but the buoyancy force become opposing so as to resist the original upward flow near the cold wall. Thus, flow reversal occurs near the cold wall for increasing values of $\lambda$. The larger the values of $\lambda<0$, stronger the downward velocity. For large values of $\lambda<0$, the flow reversal is found near the right wall as shown in Figure 2. It is also observed from Figure 2 that the asymptote in the narrower passage is faster than in the wider passage. 
For $\lambda>0$, in the wider passage, the maximum velocity point moves towards the hot side and for $\lambda<0$, the maximum velocity point moves towards the cold side. While $\lambda>0$ or $\lambda<0$, the maximum velocity point is nearly in the middle of the passage for the narrower passage. When the baffle is in the middle of the channel, similar profiles are obtained in the two passages for both $\lambda>0$ and $\lambda<0$.

The effect of buoyancy-to-inertia force $\lambda$ on the microrotation velocity for various baffle position are displayed in Figure 3 with $K=2$. The larger the values of $\lambda>0$, the stronger the downward velocity, whereas for $\lambda<0$, larger the values of $\lambda$, the stronger the upward microrotation velocity. This result is in contradiction with the velocity. Maximum and minimum point of the microrotation velocity is found near hotter wall for baffle position at 0.2 and near the cold wall for baffle position at 0.8 . When the baffle position is in the middle of the channel, there are two maximum/minimum points of the microrotation velocity profiles. It is interesting to observe that the microrotation velocity is not distorted for variations of $\lambda$ in narrower passage and its effect is highly significant in the wider passage.

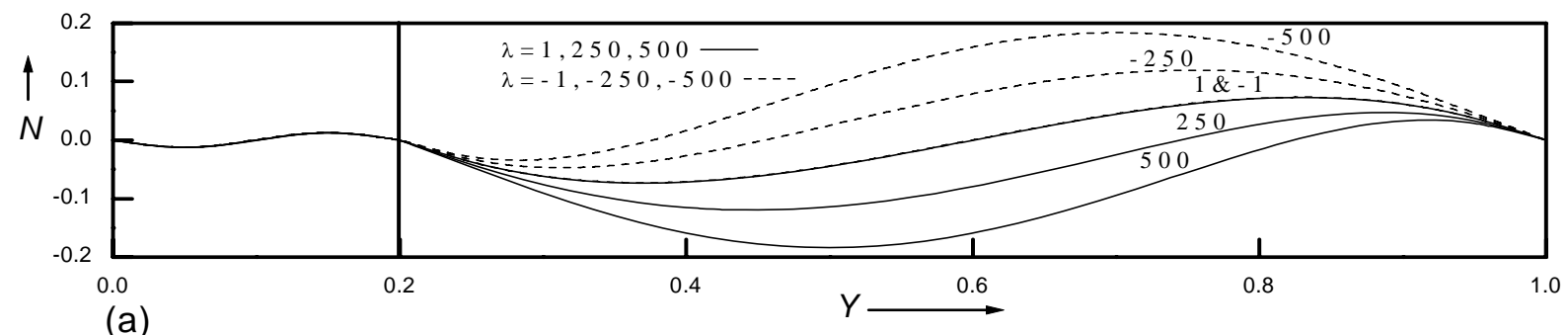

(a)
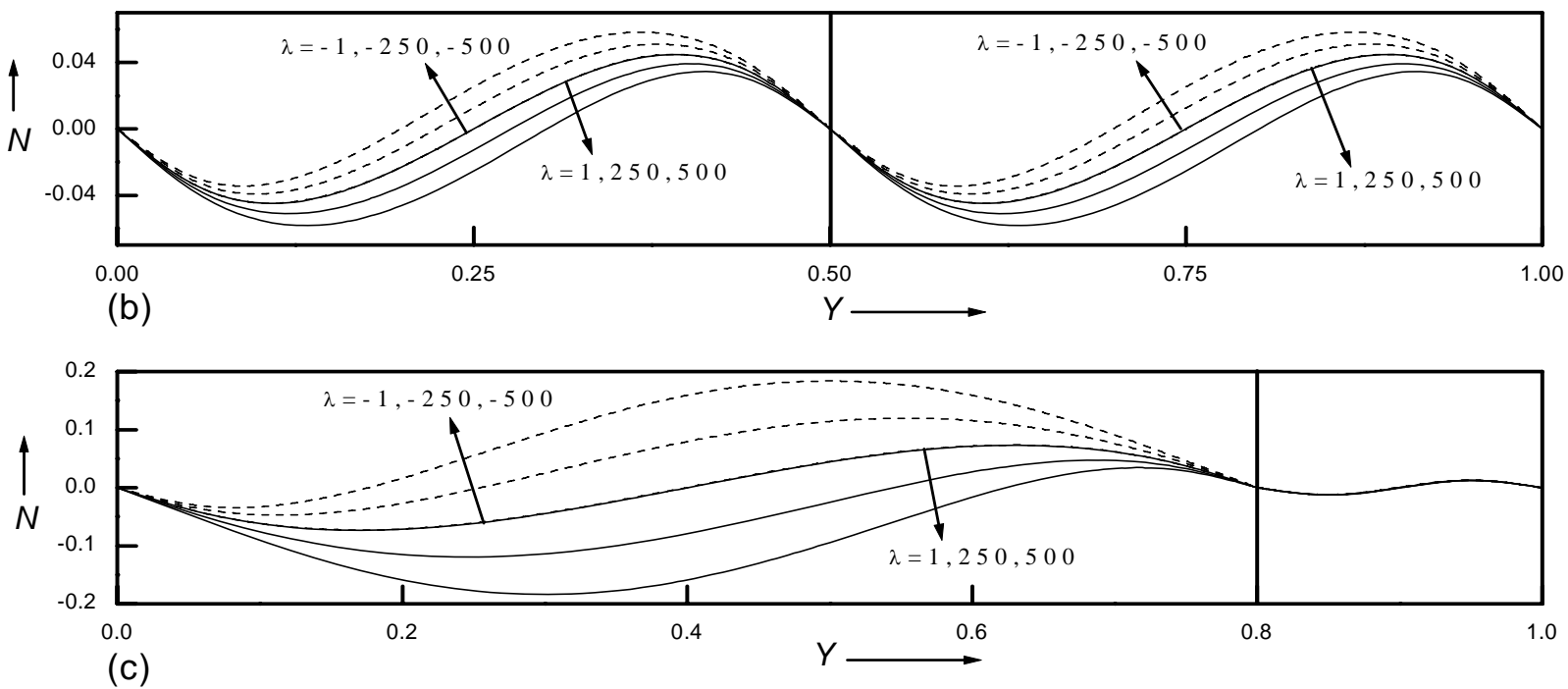

Figure 3: Microrotation velocity profiles for varies $\mathrm{Y}^{*}$ with $\lambda= \pm(1,250,500)$ and $\mathrm{K}=2$, a) $\mathrm{Y}^{*}=0.2$, b) $\mathrm{Y}^{*}=0.5$ and c) $\mathrm{Y}^{*}=0.8$.

The effect of material parameter $K$ on the microrotation velocity at three different baffle position $\left(Y^{*}=0.2,0.5,0.8\right)$ with $\lambda=50$ is shown in Figure 4. When the baffle position is at $Y^{*}=0.2$, the microrotation velocity decreases from $Y=0.2$ to 0.5 and reverses its direction from 0.5 and onwards. When the baffle position is at 0.5 , the microrotation velocity decreases from $Y=0$ to 0.25 , reverses its direction from 0.25 to 0.5 , again decreases from 0.5 to 0.75 and reverses its direction from 0.75 to 1.0 . For the baffle position at $Y^{*}=0.8$, the microrotation velocity decreases from $Y=0$ to 0.4 and reverses its direction from 0.4 and onwards. The effect of the material parameter $K$ on microrotation velocity is insignificant in the narrower passage.

The effect of material parameter on the velocity at three different baffle position $\left(Y^{*}=0.2,0.5,0.8\right)$ with $\lambda=50$ is shown in Table-1(a,b). It is observed that the effect of material parameter $K$ is very sensitive for different position of the baffle. 

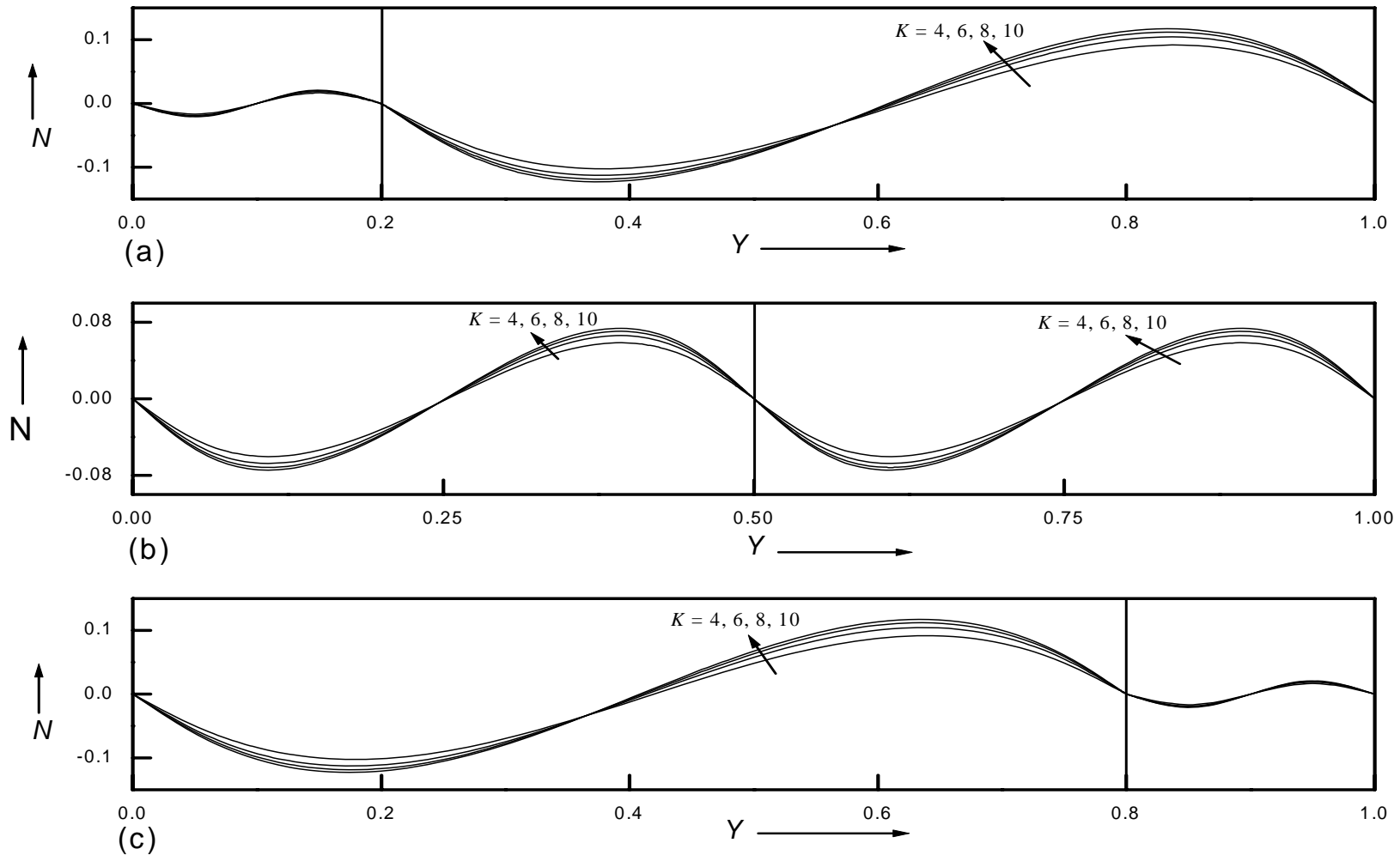

Figure 4: Microrotation velocity for varies $\mathrm{Y}^{*}$ with $K=4,6,8,10$ and $\lambda=50$, a) $Y^{*}=0.2$, b) $Y^{*}=0.5$ and c) $Y^{*}=0.8$.

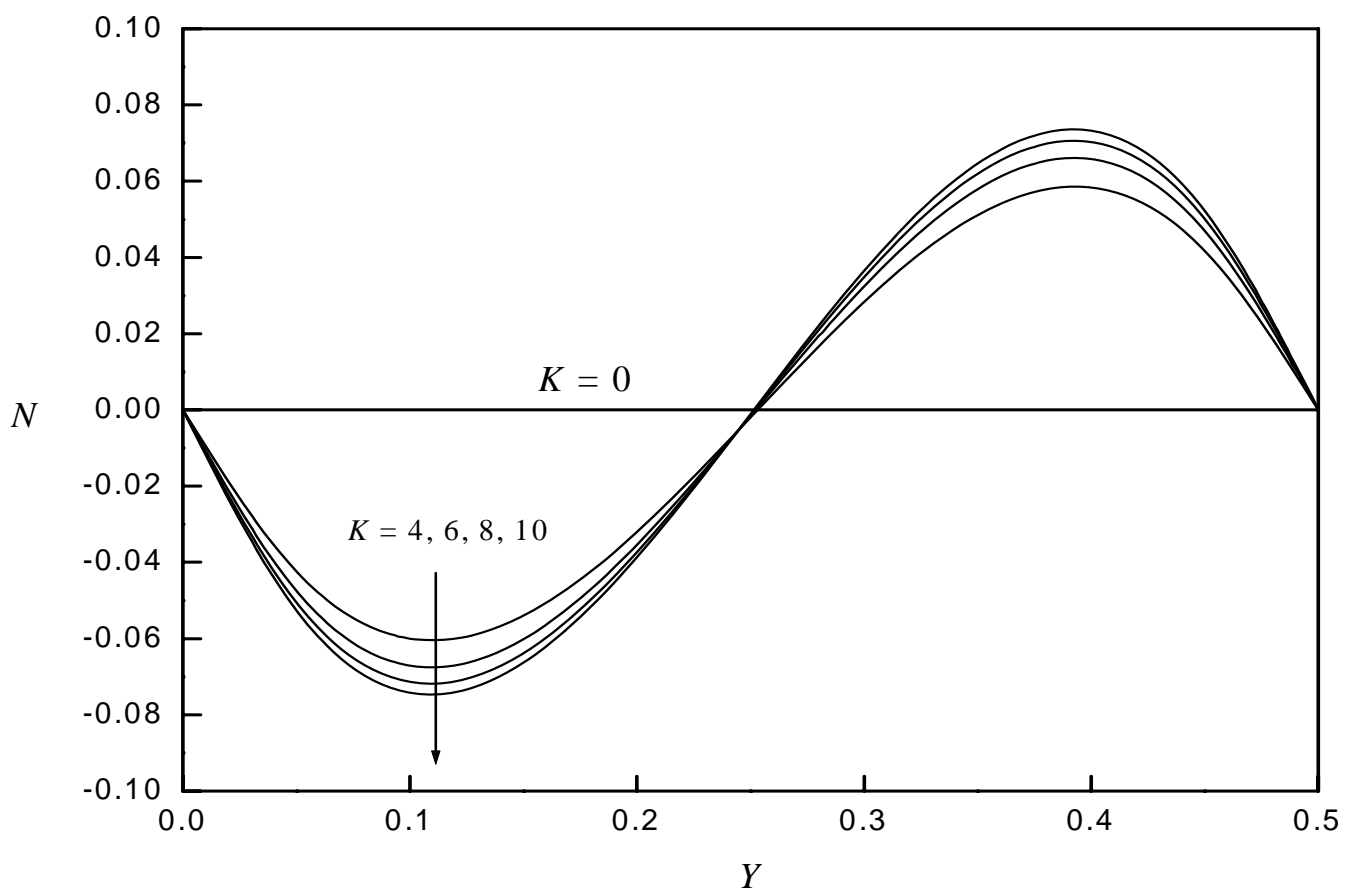

Figure 4b: Effect of material parameter $K$ on microrotation velocity for strem-1. 
Table 1a. Values of velocity for different material parameter and baffle positions with $\lambda=50$.

\begin{tabular}{|l|l|l|l|l|l|l|l|l|}
\hline & \multicolumn{5}{|c|}{$Y^{*}=0.2$} & \multicolumn{5}{c|}{$Y^{*}=0.5$} \\
\hline \multicolumn{1}{|c|}{$K=4$} & \multicolumn{1}{|c|}{$K=6$} & \multicolumn{1}{|c|}{$K=8$} & \multicolumn{1}{|c|}{$K=10$} & \multicolumn{1}{c|}{$K=4$} & \multicolumn{1}{c|}{$K=6$} & \multicolumn{1}{c|}{$K=8$} & $K=10$ \\
\hline 0 & 0 & 0 & 0 & 0 & 0 & 0 & 0 & 0 \\
\hline 0.1 & 1.50027 & 1.50032 & 1.50035 & 1.50038 & 0.9491 & 0.95179 & 0.95328 & 0.95423 \\
\hline 0.2 & 0 & 0 & 0 & 0 & 1.43622 & 1.43791 & 1.43887 & 1.43949 \\
\hline 0.3 & 0.61753 & 0.62695 & 0.63216 & 0.63546 & 1.4463 & 1.44512 & 1.44448 & 1.44408 \\
\hline 0.4 & 1.0836 & 1.09497 & 1.1013 & 1.10534 & 0.96922 & 0.96619 & 0.96449 & 0.9634 \\
\hline 0.5 & 1.38345 & 1.39119 & 1.39554 & 1.39833 & 0 & 0 & 0 & 0 \\
\hline 0.6 & 1.50409 & 1.50491 & 1.50542 & 1.50577 & 0.9491 & 0.95179 & 0.95328 & 0.95423 \\
\hline 0.7 & 1.43433 & 1.42765 & 1.42396 & 1.42161 & 1.43622 & 1.43791 & 1.43887 & 1.43949 \\
\hline 0.8 & 1.16488 & 1.1532 & 1.14668 & 1.14251 & 1.4463 & 1.44512 & 1.44448 & 1.44408 \\
\hline 0.9 & 0.68847 & 0.67774 & 0.67173 & 0.66787 & 0.96922 & 0.96619 & 0.96449 & 0.9634 \\
\hline 1 & 0 & 0 & 0 & 0 & 0 & 0 & 0 & 0 \\
\hline
\end{tabular}

Table 1b. Values of velocity for different material parameter and baffle positions with $\lambda=50$.

\begin{tabular}{|l|l|l|l|l|}
\hline & \multicolumn{4}{|c|}{$Y^{*}=0.8$} \\
\hline$Y$ & \multicolumn{1}{|c|}{$K=4$} & \multicolumn{1}{|c|}{$K=6$} & \multicolumn{1}{|c|}{$K=8$} & $K=10$ \\
\hline 0 & 0 & 0 & 0 & 0 \\
\hline 0.1 & 0.61753 & 0.62695 & 0.63216 & 0.63546 \\
\hline 0.2 & 1.0836 & 1.09497 & 1.1013 & 1.10534 \\
\hline 0.3 & 1.38345 & 1.39119 & 1.39554 & 1.39833 \\
\hline 0.4 & 1.50409 & 1.50491 & 1.50542 & 1.50577 \\
\hline 0.5 & 1.43433 & 1.42765 & 1.42396 & 1.42161 \\
\hline 0.6 & 1.16488 & 1.1532 & 1.14668 & 1.14251 \\
\hline 0.7 & 0.68847 & 0.67774 & 0.67173 & 0.66787 \\
\hline 0.8 & 0 & 0 & 0 & 0 \\
\hline 0.9 & 1.50027 & 1.50032 & 1.50035 & 1.50038 \\
\hline 1 & 0 & 0 & 0 & 0 \\
\hline
\end{tabular}

In the absence of material parameter $K$, Eqs. (8) to (10) reduce to the Eqs. (5) and (6) of Salah El-Din (2002) in the absence of viscous dissipation. For fixed value of material parameter $K$, the values for velocity at different baffle position is tabulated and shown in Table-2. It is seen that there is a good agreement between the present model and Salah El-Din (2002) model.

To further justify the present model, the effect of material parameter $K$ on the microrotation velocity when the baffle is in the centre of the channel is compared with Chamkha et al. (2002). The present result and the result of Chamkha et al. (2002) show the similar effect.

Table 2. Comparison of present model with Salah El-Din (2002) for $\lambda=1$.

\begin{tabular}{|l|l|l|l|l|l|l|l|l|l|l|l|}
\hline & \multicolumn{4}{|c|}{$Y^{*}=0.2$} & \multicolumn{4}{c|}{$Y^{*}=0.5$} & \multicolumn{4}{c|}{$Y^{*}=0.8$} \\
\hline$Y$ & $\begin{array}{c}\text { Present } \\
K=2\end{array}$ & $\begin{array}{c}\text { Salah El- } \\
\text { Din (2002) }\end{array}$ & $\begin{array}{c}\text { \% of } \\
\text { error }\end{array}$ & $Y$ & $\begin{array}{c}\text { Present } \\
K=2\end{array}$ & $\begin{array}{c}\text { Salah El- } \\
\text { Din (2002) }\end{array}$ & $\begin{array}{c}\text { \% of } \\
\text { error }\end{array}$ & $Y$ & $\begin{array}{c}\text { Present } \\
K=2\end{array}$ & $\begin{array}{c}\text { Salah El- } \\
\text { Din (2002) }\end{array}$ & $\begin{array}{c}\text { \% of } \\
\text { error }\end{array}$ \\
\hline 0 & 0 & 0 & 0.00 & 0 & 0 & 0 & 0.00 & 0 & 0 & 0 & 0.00 \\
\hline 0.05 & 1.12495 & 1.12494 & 0.00 & 0.05 & 0.53944 & 0.53925 & 0.02 & 0.05 & 0.35058 & 0.34937 & 0.12 \\
\hline 0.1 & 1.50017 & 1.5 & 0.02 & 0.1 & 0.95981 & 0.959 & 0.08 & 0.1 & 0.65538 & 0.65275 & 0.26 \\
\hline 0.15 & 1.12499 & 1.12506 & 0.01 & 0.15 & 1.26047 & 1.25913 & 0.13 & 0.15 & 0.91393 & 0.91 & 0.39 \\
\hline 0.2 & 0 & 0 & 0.00 & 0.2 & 1.44096 & 1.4395 & 0.15 & 0.2 & 1.12587 & 1.121 & 0.49 \\
\hline 0.2 & 0 & 0 & 0.00 & 0.25 & 1.50103 & 1.5 & 0.10 & 0.25 & 1.29088 & 1.28562 & 0.53 \\
\hline 0.25 & 0.34911 & 0.34937 & 0.03 & 0.3 & 1.44062 & 1.4405 & 0.01 & 0.3 & 1.40876 & 1.40375 & 0.50 \\
\hline 0.3 & 0.65302 & 0.65275 & 0.03 & 0.35 & 1.25988 & 1.26087 & 0.10 & 0.35 & 1.47935 & 1.47525 & 0.41 \\
\hline 0.35 & 0.9112 & 0.91 & 0.12 & 0.4 & 0.95914 & 0.961 & 0.19 & 0.4 & 1.50258 & 1.5 & 0.26 \\
\hline 0.4 & 1.12317 & 1.121 & 0.22 & 0.45 & 0.53893 & 0.54075 & 0.18 & 0.45 & 1.47846 & 1.47787 & 0.06 \\
\hline 0.45 & 1.28857 & 1.28562 & 0.30 & 0.5 & 0 & 0 & 0.00 & 0.5 & 1.40707 & 1.40875 & 0.17 \\
\hline 0.5 & 1.40707 & 1.40375 & 0.33 & 0.5 & 0 & 0 & 0.00 & 0.55 & 1.28857 & 1.2925 & 0.39 \\
\hline
\end{tabular}


Table 2 (cont'd). Comparison of present model with Salah El-Din (2002) for $\lambda=1$.

\begin{tabular}{|l|l|l|l|l|l|l|l|l|l|l|l|}
\hline & \multicolumn{4}{|c|}{$Y^{*}=0.2$} & \multicolumn{4}{c|}{$Y^{*}=0.5$} & \multicolumn{4}{c|}{$Y^{*}=0.8$} \\
\hline$Y$ & $\begin{array}{c}\text { Present } \\
K=2\end{array}$ & $\begin{array}{c}\text { Salah El- } \\
\text { Din (2002) }\end{array}$ & $\begin{array}{c}\text { \% of } \\
\text { error }\end{array}$ & $Y$ & $\begin{array}{c}\text { Present } \\
K=2\end{array}$ & $\begin{array}{c}\text { Salah El- } \\
\text { Din (2002) }\end{array}$ & $\begin{array}{c}\text { \% of } \\
\text { error }\end{array}$ & $Y$ & $\begin{array}{c}\text { Present } \\
K=2\end{array}$ & $\begin{array}{c}\text { Salah El- } \\
\text { Din (2002) }\end{array}$ & $\begin{array}{c}\text { \% of } \\
\text { error }\end{array}$ \\
\hline 0.55 & 1.47846 & 1.47525 & 0.32 & 0.55 & 0.53944 & 0.53925 & 0.02 & 0.6 & 1.12317 & 1.129 & 0.58 \\
\hline 0.6 & 1.50258 & 1.5 & 0.26 & 0.6 & 0.95981 & 0.959 & 0.08 & 0.65 & 0.9112 & 0.91812 & 0.69 \\
\hline 0.65 & 1.47935 & 1.47788 & 0.15 & 0.65 & 1.26047 & 1.25913 & 0.13 & 0.7 & 0.65302 & 0.65975 & 0.67 \\
\hline 0.7 & 1.40876 & 1.40875 & 0.00 & 0.7 & 1.44096 & 1.4395 & 0.15 & 0.75 & 0.34911 & 0.35375 & 0.46 \\
\hline 0.75 & 1.29088 & 1.2925 & 0.16 & 0.75 & 1.50103 & 1.5 & 0.10 & 0.8 & 0 & 0 & 0.00 \\
\hline 0.8 & 1.12587 & 1.129 & 0.31 & 0.8 & 1.44062 & 1.4405 & 0.01 & 0.8 & 0 & 0 & 0.00 \\
\hline 0.85 & 0.91393 & 0.91812 & 0.42 & 0.85 & 1.25988 & 1.26087 & 0.10 & 0.85 & 1.12499 & 1.12494 & 0.00 \\
\hline 0.9 & 0.65538 & 0.65975 & 0.44 & 0.9 & 0.95914 & 0.961 & 0.19 & 0.9 & 1.50017 & 1.5 & 0.02 \\
\hline 0.95 & 0.35058 & 0.35375 & 0.32 & 0.95 & 0.53893 & 0.54075 & 0.18 & 0.95 & 1.12495 & 1.12506 & 0.01 \\
\hline 1 & 0 & 0 & 0.00 & 1 & 0 & 0 & 0.00 & 1 & 0 & 0 & 0.00 \\
\hline
\end{tabular}

\section{Conclusions}

An analytical investigation of the laminar mixed convection in a vertical double passage channel filled with micropolar fluid has been presented. The channel is divided into two passages by means of a perfectively conductive, thin-plane baffle. The development of the velocity and microrotation velocity profiles in the channel has been presented. The parametric study has been carried out to evaluate the influence of buoyancy-to-inertia and material parameter on the velocity and microrotation velocity at different positions of the baffle.

It has been concluded that

1. The presence of the baffle leads to the maxima and minima of velocity and microrotation velocity profiles.

2. For buoyancy assisted flow i.e., for $\lambda>0$, the maxima of velocity is at the right wall. For buoyancy opposing flow i.e., $\lambda<0$, the maxima of velocity is at the left wall. The opposite effect is observed on microrotation velocity.

3. The effect of material parameter is insignificant on the velocity. The effect of material parameter on the microrotation velocity depends on the length of the channel.

4. Fixing the material parameter, the values of velocity at different baffle positions are close to the values obtained by Salah El-Din (2002).

5. The effects of material parameter on the microrotation velocity are similar to the results obtained by Chamkha et al. (2002) when the baffle is placed in the middle of the channel.

\section{Appendix}

$$
\begin{aligned}
& \tau=\frac{2 K}{K+1}, l_{1}=\frac{1}{K+2}, l_{2}=\frac{\lambda}{2(2+K)}, l_{3}=\frac{K+1}{K+2}, l_{41}=-l_{2}, l_{42}=-l_{1}, l_{51}=\frac{l_{3} \lambda}{2 K}, l_{52}=-l_{3}, d_{11}=-l_{51}, d_{12}=-l_{52}, \\
& d_{21}=\frac{-d_{11} \cosh \left(\sqrt{\tau} Y^{*}\right)-l_{2} Y^{* 2}-l_{41} Y^{*}-l_{51}}{\sinh \left(\sqrt{\tau} Y^{*}\right)}, d_{22}=\frac{-d_{12} \cosh \left(\sqrt{\tau} Y^{*}\right)-l_{52}}{\sinh \left(\sqrt{\tau} Y^{*}\right)}, d_{23}=\frac{-l_{42} Y^{*}}{\sinh \left(\sqrt{\tau} Y^{*}\right)}, f_{11}=\frac{-K d_{11}}{(1+K) \sqrt{\tau}}, f_{42}=\frac{1-K l_{42}}{2(1+K)}, \\
& f_{12}=\frac{-K d_{12}}{(1+K) \sqrt{\tau}}, f_{21}=\frac{-K d_{21}}{(1+K) \sqrt{\tau}}, f_{22}=\frac{-K d_{22}}{(1+K) \sqrt{\tau}}, f_{23}=\frac{-K d_{23}}{(1+K) \sqrt{\tau}}, f_{3}=\frac{-K l_{2}}{3(1+K)}-\frac{\lambda}{6(1+K)}, f_{41}=\frac{\lambda}{4(1+K)}-\frac{K l_{41}}{2(1+K)}, \\
& c_{21}=-f_{21}, c_{22}=-f_{22}, b_{23}=-f_{23}, \quad c_{11}=\frac{-1}{Y^{*}}\left(f_{11} \sinh \left(\sqrt{\tau} Y^{*}\right)+f_{21} \cosh \left(\sqrt{\tau} Y^{*}\right)+f_{3} Y^{* 3}+f_{41} Y^{* 2}+c_{21}\right), \\
& c_{12}=\frac{-1}{Y^{*}}\left(f_{12} \sinh \left(\sqrt{\tau} Y^{*}\right)+f_{22} \cosh \left(\sqrt{\tau} Y^{*}\right)+c_{22}\right), \quad c_{13}=\frac{-1}{Y^{*}}\left(f_{23} \cosh \left(\sqrt{\tau} Y^{*}\right)+f_{42} Y^{* 2}+c_{23}\right), \quad A_{11}=\frac{-K l_{51}-c_{11}(1+K)}{(1+K) c_{12}+K l_{52}-(1+K)}, \\
& A_{12}=\frac{-c_{13}(1+K)}{(1+K) c_{12}+K l_{52}-(1+K)}, \quad l_{4}=l_{41}+\gamma_{1} l_{42}, \quad l_{5}=l_{51}+A_{1} l_{52}, \quad d_{2}=d_{21}+A_{1} d_{22}+\gamma_{1} b_{23}, \quad d_{1}=d_{11}+A_{1} d_{12}, \quad f_{1}=f_{11}+A_{1} f_{12}, \\
& f_{2}=f_{21}+A_{1} f_{22}+\gamma_{1} f_{23}, f_{4}=f_{41}+\gamma_{1} f_{42}, c_{2}=c_{21}+A_{1} c_{22}+\gamma_{1} c_{23}, \quad c_{1}=c_{11}+A_{1} c_{12}+\gamma_{1} c_{13}, A_{1}=A_{11}+\gamma_{1} A_{12},
\end{aligned}
$$




$$
\begin{aligned}
& l_{61}=-l_{2}, \quad l_{62}=-l_{1}, \quad l_{71}=\frac{l_{3} \lambda}{2 K}, \quad l_{72}=-l_{3}, \quad \operatorname{Dr}=\sinh (\sqrt{\tau}) \cosh \left(\sqrt{\tau} Y^{*}\right)-\sinh \left(\sqrt{\tau} Y^{*}\right) \cosh (\sqrt{\tau}), \\
& d_{41}=\frac{1}{\operatorname{Dr}}\left(l_{2}\left(Y^{* 2} \cosh (\sqrt{\tau})-\cosh \left(\sqrt{\tau} Y^{*}\right)\right)+l_{61}\left(Y^{*} \cosh (\sqrt{\tau})-\cosh \left(\sqrt{\tau} Y^{*}\right)\right)+l_{71}\left(\cosh (\sqrt{\tau})-\cosh \left(\sqrt{\tau} Y^{*}\right)\right)\right) \text {, } \\
& d_{42}=\frac{l_{71}}{D r}\left(\cosh (\sqrt{\tau})-\cosh \left(\sqrt{\tau} Y^{*}\right)\right), \quad d_{43}=\frac{l_{62}}{\operatorname{Dr}}\left(Y^{*} \cosh (\sqrt{\tau})-\cosh \left(\sqrt{\tau} Y^{*}\right)\right), \quad d_{31}=\frac{-d_{41} \sinh (\sqrt{\tau})-l_{2}-l_{61}-l_{71}}{\cosh (\sqrt{\tau})}, \\
& d_{32}=\frac{-d_{42} \sinh (\sqrt{\tau})-l_{72}}{\cosh (\sqrt{\tau})}, d_{31}=\frac{-d_{43} \sinh (\sqrt{\tau})-l_{62}}{\cosh (\sqrt{\tau})}, f_{51}=\frac{-K d_{31}}{(1+K) \sqrt{\tau}}, f_{52}=\frac{-K d_{32}}{(1+K) \sqrt{\tau}}, f_{53}=\frac{-K d_{33}}{(1+K) \sqrt{\tau}}, f_{61}=\frac{-K d_{41}}{(1+K) \sqrt{\tau}} \text {, } \\
& f_{62}=\frac{-K d_{42}}{(1+K) \sqrt{\tau}}, \quad f_{63}=\frac{-K d_{43}}{(1+K) \sqrt{\tau}}, \quad f_{7}=\frac{-K l_{2}}{3(1+K)}-\frac{\lambda}{6(1+K)}, \quad f_{81}=\frac{\lambda}{4(1+K)}-\frac{K l_{61}}{2(1+K)}, \quad f_{82}=\frac{1-K l_{62}}{2(1+K)} \text {, } \\
& c_{31}=\frac{-1}{1-Y^{*}}\left(f_{51}\left(\sinh (\sqrt{\tau})-\sinh \left(\sqrt{\tau} Y^{*}\right)\right)+f_{61}\left(\cosh (\sqrt{\tau})-\cosh \left(\sqrt{\tau} Y^{*}\right)\right)+l_{71}\left(1-Y^{* 3}\right)+f_{81}\left(1-Y^{* 2}\right)\right) \text {, } \\
& c_{32}=\frac{-1}{1-Y^{*}}\left(f_{52}\left(\sinh (\sqrt{\tau})-\sinh \left(\sqrt{\tau} Y^{*}\right)\right)+f_{62}\left(\cosh (\sqrt{\tau})-\cosh \left(\sqrt{\tau} Y^{*}\right)\right)\right) \text {, } \\
& c_{33}=\frac{-1}{1-Y^{*}}\left(f_{53}\left(\sinh (\sqrt{\tau})-\sinh \left(\sqrt{\tau} Y^{*}\right)\right)+f_{63}\left(\cosh (\sqrt{\tau})-\cosh \left(\sqrt{\tau} Y^{*}\right)\right)+f_{82}\left(1-Y^{* 2}\right)\right), \\
& c_{41}=-c_{31}-f_{51} \sinh (\sqrt{\tau})-f_{61} \cosh (\sqrt{\tau})-f_{7}-f_{81}, \quad c_{42}=-f_{52} \sinh \left(\sqrt{\tau} Y^{*}\right)-f_{62} \cosh \left(\sqrt{\tau} Y^{*}\right)-c_{32} \text {, } \\
& c_{43}=-f_{53} \cosh (\sqrt{\tau})-f_{63} \cosh \left(\sqrt{\tau} Y^{*}\right)-c_{32}-f_{82}, A_{21}=\frac{-K l_{71}-c_{31}(1+K)}{(1+K) c_{32}+K l_{72}-(1+K)}, A_{22}=\frac{-c_{33}(1+K)}{(1+K) c_{32}+K l_{72}-(1+K)} l_{6}=l_{61}+\gamma_{2} l_{62} \text {, } \\
& l_{7}=l_{71}+A_{2} l_{72}, \quad d_{4}=d_{41}+A_{2} d_{42}+\gamma_{2} b_{43}, \quad d_{3}=d_{31}+A_{2} d_{32}+\gamma_{2} b_{33}, \quad f_{5}=f_{51}+A_{2} f_{52}+\gamma_{2} f_{53}, \quad f_{6}=f_{61}+A_{2} f_{62}+\gamma_{2} f_{63}, \\
& f_{8}=f_{81}+\gamma_{2} f_{82}, c_{3}=c_{31}+A_{2} c_{32}+\gamma_{2} c_{33}, c_{4}=c_{41}+A_{2} c_{42}+\gamma_{2} c_{43} \text {. }
\end{aligned}
$$

\section{Nomenclature}

$b \quad$ channel width $(\mathrm{m})$

$b^{*} \quad$ width of stream $(\mathrm{m})$

$C_{p} \quad$ specific heat at constant pressure $\left(\mathrm{kJ} \mathrm{kg}^{-1} \mathrm{~K}^{-1}\right)$

g gravitational acceleration $\left(\mathrm{m} \mathrm{s}^{-2}\right)$

Gr Grashof number

j microinertia density $\left(\mathrm{kg} \mathrm{m}^{-3}\right)$

$\bar{N} \quad$ microrotation velocity $\left(\mathrm{m} \mathrm{s}^{-1}\right)$

$N \quad$ dimensionless microrotation velocity

$K \quad$ non-dimensional material parameter

$m \quad$ viscosity ratio

$p \quad$ pressure $\left(\mathrm{Nm}^{-2}\right)$

$P \quad$ dimensionless pressure

Re Reynolds number

$T$ temperature (K)

$T_{r} \quad$ reference temperature $(\mathrm{K})$

$u \quad$ axial velocity $\left(\mathrm{m} \mathrm{s}^{-1}\right)$

$U \quad$ dimensionless velocity

Wp pressure gradient ratio

$x \quad$ axial coordinate $(\mathrm{m})$ 
$\begin{array}{ll}y & \text { transverse coordinate }(\mathrm{m}) \\ Y^{*} & \text { baffle position }\end{array}$

\section{Greek symbols}

$\begin{array}{ll}\alpha & \text { spin-gradient viscosity }\left(\mathrm{kg} \mathrm{m}^{-1} \mathrm{~s}^{-1}\right) \\ \beta & \text { volumetric coefficient of thermal expansion }\left(\mathrm{K}^{-1}\right) \\ \gamma & \text { pressure gradient parameter } \\ \kappa & \text { material parameter } \\ \theta & \text { dimensionless temperature } \\ \mu & \text { dynamic viscosity }\left(\mathrm{kg} \mathrm{m}^{-1} \mathrm{~s}^{-1}\right) \\ v & \text { kinematics viscosity } \\ \rho & \text { density }\left(\mathrm{kg} \mathrm{m}^{-3}\right)\end{array}$

\begin{tabular}{ll}
\multicolumn{2}{l}{ Subscripts } \\
$b$ & bulk \\
$c$ & cold wall \\
$h$ & hot wall \\
$r$ & reference \\
1 & value in stream 1 \\
2 & value in stream 2
\end{tabular}

\section{Acknowledgments}

Author would like to thank UGC-New Delhi for the financial support under UGC-Major Research Project.

\section{References}

Abdul Hakeem, A.K., Saravanan, S, and Kandaswamy, P., 2008. Buoyancy convection in a square cavity with mutually orthogonal heat generating baffles, Int. J. Heat and Fluid Flow, Vol. 29, pp. 1164-1173.

Ahmadi, G., 1976. Int. J. Engng. Sci., Vol. 14, pp. 639.

Ali J. Chamkha, Grosan, T. and Pop, I., 2002. Fully developed free convection of a micropolar fluid in a vertical channel, Int. Comm. Heat Mass Transfer, Vol. 29, pp. 1119-1127.

Anwar Bég, O., Bhargava R., and Rashidi, M.M., 2011. Numerical Simulation in Micropolar Fluid Dynamics (Mathematical Modelling of Nonlinear Flows of Micropolar Fluids) Lambert Academic Press, Germany. Pp. 296.

Ariman, T., Turk, M.A. and Sylvester, N.D., 1974. Review article-applications of microcontinuum fluid mechanics, Int. J. Engng. Sci., Vol. 12, pp. 273-293.

Barletta, A., 1998. Laminar mixed convection with viscous dissipation in a vertical channel, Int. J. Heat Mass Transfer, Vol. 41, pp. 3501-3513.

Cheng, C.Y., 2006. Fully developed natural convection heat and mass transfer of a micropolar fluid in a vertical channel with asymmetric wall temperatures and concentrations, Int. Com. in Heat and Mass Transfer, Vol. 33, pp. 627-635.

Cheng, C.Y., 2008. Natural convection heat and mass transfer from a sphere in micropolar fluids with constant wall temperature and concentration, Int. Com. In Heat and Mass Transfer, Vol. 35, pp. 750-755.

Dutta, P. and Dutta, S., 1998. Effect of baffle size perforation and orientation on internal heat transfer enhancement, Int. J. Heat Mass Transfer, Vol. 41, pp. 3005-3013.

Dutta, P. and Hossain, A., 2005. Internal cooling augmentation in rectangular channel using two inclined baffles, Int. J. Heat Fluid Flow, Vol. 26, pp. 223-232.

El-Hakiem, M.A., 2004. Natural convection in a micropolar fluid with thermal dispersion and internal heat generation, Int. Comm. Heat Mass Transfer, Vol. 31, pp. 1177-1186.

Eringen, A.C., 1964. Simple microfluids, Int. J. Engng. Sci., Vol. 2, pp. 205-217.

Eringen, A.C., 1966. Theory of micropolar fluids, J. Math. Mech., Vol. 16, pp. 1-18.

Eringen, A.C., 2001. Microcontinuum field theories II: Fluent Media, Springer-Verlog, New York, Inc.

Howes, T. and Shardlow, J., 1997. Simulation of mixing in unsteady flow through a periodically obstructed channel, Chem. Engng. Sci., Vol. 52, pp. 1215-1225. 
Howes, T., Mackley, M.R. and Roberts, E.P.L., 1991. The simulation of chaotic mixing and dispersion for periodic flows in baffled channels, Chem. Engng. Sci., Vol. 46, pp. 1669-1677.

Hoyt, J.W. and Fabula, A.G., 1964. The effect of additives on fluid friction, US Naval Ordinance Test Station Report.

Kumar, L., Singh, B., Lokesh Kumar, and Bhargava, 2011. Finite element solution of MHD flow of micropolar fluid towards a stagnation point on a vertical stretching sheet, Int. J. Appl. Math. Mech., Vol. 7(3), pp. 14-30.

Mousavi, S.S., and Hooman, K., 2006. Heat and fluid flow in entrance region of a channel with staggered baffles, Energy Conservation and Management, Vol. 47, pp. 2011-2019.

Popautsky, I., Brazzle, J. Ameel, T. and Frazier, A.B., 1999. Laminar fluid behavior in microchannels using micropolar fluid theory, Sesors Actators A Physical, Vol. 73, pp. 101-108.

Prathap Kumar, J., Umavathi, J.C., Ali J. Chamkha, and Ioan Pop, 2010. Fully-developed free-convective flow of micropolar and viscous fluids in a vertical channel, Applied Mathematical Modelling, Vol. 34, pp. 1175-1186.

Prathap Kumar, J., Umavathi, J.C., Ali J. Chamkha and Prema, H, 2011. Free convection in a vertical double passage wavy channel filled with a Walters fluid (model B'), Int. J. Energy \& Technology, Vol. 3(2), pp. 1-13.

Prathap Kumar, J., Umavathi, J.C., and Prema, H., 2011. Free convection of Walter's fluid flow in a vertical double-passage wavy channel with heat source, Int. J. Eng. Sci. Techno., Vol. 3(1), pp. 136-165.

Nasiruddin, M.H, and Siddiqui, K., 2007. Heat transfer augmentation in a heat exchanger tube using a baffle, Int. J. Heat and Fluid Flow, Vol. 28(2), pp. 318-328.

Rama Bhargava, Lokendra Kumar and Takhar, H.S., 2003. Finite element solution of mixed convection micropolar flow driven by a porous stretching sheet, Int. J. Engng. Sci., Vol. 41, pp. 2161-2178.

Rashidi, M.M., Mohimanian Pour, S.A., and Laraqi, N., 2010. A semi-analytical solution of micropolar flow in a porous channel with mass injection by using differential transform method, Nonlinear Analysis: Modelling and Control Vol. 15, pp. 341-350.

Rashidi, M.M., Mohimanian Pour, S.A., and Abbasbandy, S., 2011. Analytic approximate solutions for heat transfer of a micropolar fluid through a porous medium with radiation, Commu. Nonlinear Science and Numerical Simulation, Vol. 16, pp. 1874-1889.

Salah El-Din, M.M., 1994. Fully developed laminar convection in a vertical double-passage channel, Appl. Energy, Vol. 47, pp. 6975.

Salah El-Din, M.M., 1997. Enhancement of heat transfer in a vertical channel, in: The Second Jordanian International Conference for Mechanical Engineering, Amman, pp. 400-412.

Salah El-Din, M.M., 1998. Developing laminar convection in a vertical double-passage channel, Heat Mass Transfer, Vol. 41, pp. 3501-3513.

Salah El-Din, M.M., 2002. Effect of viscous dissipation on fully developed on fully developed laminar mixed convection in a vertical double-passage channel, Int. J. Thermal Sci., Vol. 41, pp. 253-259.

Shu, J.J., 2004. Microscale heat transfer in a free jet against a plane surface, Superlattices Microstructure, Vol. 35, pp. 645-656.

Tsan-Hui Hsu and Cha'o-Kuanf Chen, 1996. Natural convection of micropolar fluids in a rectangular enclosure, Int. J. Engng. Sci., Vol. 34, pp. 407-415.

Umavathi, J.C., Prathap Kumar, J. and Chamkha, A. J., 2008. Flow and heat transfer of a micropolar fluid sandwiched between viscous fluid layers, Can. J. Physics, Vol. 86, pp. 1-13.

Zanchini, E., 1998. Effect of viscous dissipation on mixed convection in a vertical channel with boundary conditions of the third kind, Int. J. Heat and Mass Transfer, Vol. 41, pp. 3949-3959.

\section{Biographical notes}

J. C. Umavathi received Ph. D degree from Gulbarga University Gulbarga India in 1992. She is a Professor in the department of Mathematics, Gulbarga University, Gulbarga, Karnataka, India. Her research interest includes heat and mass transfer of multiple (Newtonian and non-Newtonian) fluids through channels and rectangular ducts, numerical techniques using Finite differences and Runge-Kutta Gill method, magnetohydradynamics, flow through porous media. She has published more than 80 papers in referred International journals. She has also presented more than 25 research articles in National and International conferences. She is currently dealing with few projects sponsored by Government of India.

Received November 2011

Accepted May 2012

Final acceptance in revised form May 2012 